\title{
Ionic Strength Responsive Sulfonated Polystyrene Opals
}

\author{
Luca Nucara, ${ }^{*},+\ddagger$ Vincenzo Piazza, ${ }^{\S}$ Francesco Greco, ${ }^{*}, \ddagger$ Valentina Robbiano, $"$ Valentina Cappello, \\ Mauro Gemmi, ${ }^{\S}$ Franco Cacialli,, and Virgilio Mattoli* ${ }^{*}+0$ \\ ${ }^{\dagger}$ The BioRobotics Institute, Scuola Superiore Sant’Anna, Viale Rinaldo Piaggio 34, Pontedera (PI) 56025, Italy \\ ${ }^{\ddagger}$ Center for Micro-BioRobotics @SSSA, Istituto Italiano di Tecnologia, Viale Rinaldo Piaggio 34, Pontedera (PI) 56025, Italy \\ ${ }^{\S}$ Center for Nanotechnology Innovation @NEST, Istituto Italiano di Tecnologia, P.za San Silvestro 12, Pisa 56127, Italy \\ "Department of Physics and Astronomy and London Centre for Nanotechnology, University College London, Gower Street, \\ London WC1E 6BT, United Kingdom
}

\section{Supporting Information}

\begin{abstract}
Stimuli-responsive photonic crystals (PCs) represent an intriguing class of smart materials very promising for sensing applications. Here, selective ionic strength responsive polymeric PCs are reported. They are easily fabricated by partial sulfonation of polystyrene opals, without using toxic or expensive monomers and etching steps. The color of the resulting hydrogellike ordered structures can be continuously shifted over the entire visible range $(405-760 \mathrm{~nm})$ by changing the content of ions over an extremely wide range of concentration (from about $70 \mu \mathrm{M}$ to

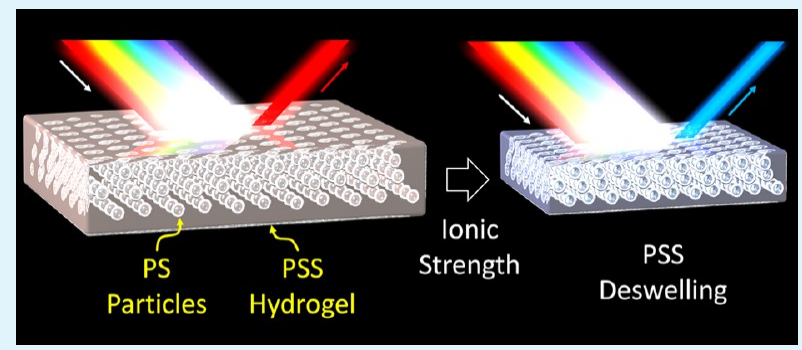
$4 \mathrm{M})$. The optical response is completely independent from $\mathrm{pH}$ and temperature, and the initial color can be fully recovered by washing the sulfonated opals with pure water. These new smart photonic materials could find important applications as ionic strength sensors for environmental monitoring as well as for healthcare screening.
\end{abstract}

KEYWORDS: ionic strength sensors, polyelectrolytes, colloids, stimuli-responsive opals, core-shell particles

\section{INTRODUCTION}

Photonic crystals (PCs) represent an important class of colored structured materials with a spatial modulation of the refractive index on a length scale comparable to the wavelength of visible light. The periodic spatial arrangement enables control of the propagation of the electromagnetic waves by creating a so-called partial or full band gap or stop band, i.e., energy intervals for which propagation is forbidden along certain or all directions. ${ }^{1}$ The use of so-called "smart materials" in the fabrication of PCs results in stimuli-responsive photonic structures, whose optical properties can be modulated by using external triggers, such as chemical, ${ }^{2-6}$ thermal, $^{7-9}$ electrical, ${ }^{10}$ magnetic, ${ }^{11,12}$ mechanical ${ }^{13,14}$ stimuli, or light. ${ }^{15-17}$ In general, the application of a stimulus can modify the PC's lattice constant and/or induce a variation of the materials' refractive indices. These effects result in a shift of the reflection band(s) and, as a consequence, in a variation of the perceived color. Stimuliresponsive PCs constitute an intriguing class of smart materials that can be applied in several technologies for sensing devices or actuation systems, among others. ${ }^{18}$

In particular, photonic structures made up of chemically responsive materials can be applied as PCs-based sensors (e.g., $\mathrm{pH}$, solvents, water content, ionic strength) whose readout is an optical response, in principle detectable by the naked eye. ${ }^{6,19}$ In this context, hydrogels are often used in responsive PCs, thanks to their versatility, ease of synthesis, and functionalization.
They can be processed to obtain opals and inverted opals and modified with functional groups which are sensible to different analytes, like heavy metals, ${ }^{20-23} \mathrm{pH}^{24,25}$ and amino acids. ${ }^{5}$

Sensing ionic strength (IS) of solutions can be useful in several applications, such as in monitoring of the salinity of sea and fresh water, in fish agriculture, during blood dialysis, and in the chemical industry. ${ }^{26}$ Although a large variety of chemically responsive PCs have been described in recent literature, very few examples focused on PCs which are selectively responsive to IS. In particular, Asher et al. presented a polyacrylamide infiltrated polystyrene colloidal crystal, which after partial hydrolysis of the amide groups exhibited an interesting IS response. $^{25}$ The peak reflection wavelength was shifted by about $250 \mathrm{~nm}$ by increasing the IS from 0 to $10 \mathrm{mM}$. More recently, by using similar materials and processes, Fenzl et al. realized a PC that can be tuned over a broader range of IS than the previous example, although the spectral variation was reduced. $^{26}$ Indeed, by increasing the IS of the surrounding solution from $10^{-5}$ to $10^{-2} \mathrm{M}$ the reflected wavelength was shifted by $\sim 100 \mathrm{~nm}$. Despite the fast response, this system is adequate for sensing the IS only at $\mathrm{pH}>5$, as the $\mathrm{PC}$ shift is strongly influenced by the $\mathrm{pH}$ under more acidic conditions.

Received: November 11, 2016

Accepted: January 12, 2017

Published: January 12, 2017 

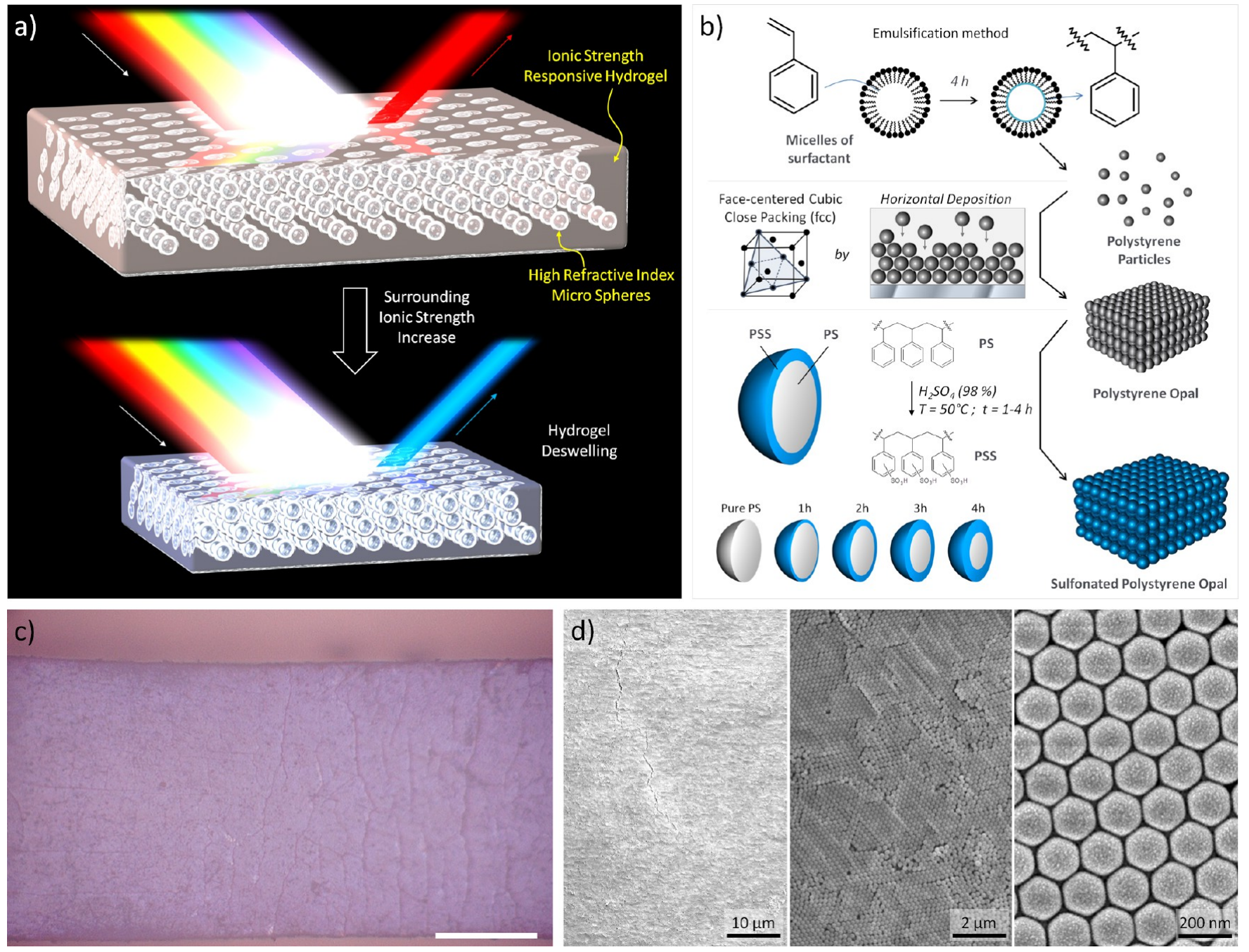

Figure 1. (a) Working mechanism of the IS responsive sulfonated PS opals. (b) Fabrication process of the sulfonated polystyrene opals. The PS particles are assembled in a photonic structure by horizontal deposition and sulfonated with concentrated sulfuric acid. Optical (c) and SEM (d) images of nonmodified PS opals. Scale bar: $200 \mu \mathrm{m}$ for (c).

An additional example was reported by Chen et al., who fabricated microgel PCs of poly ( $N$-isopropylacrylamide $)$ acrylic acid $)$ $\mathrm{P}$ (NIPAM-AAc) which were able to respond to IS and temperature. ${ }^{9}$ The color could be shifted very quickly by about $360 \mathrm{~nm}$ by increasing the IS from 10 to $667 \mathrm{mM}$, although the small size of the crystals (few micrometers) prevents the application of this material as a sensor. The cited PCs examples exhibited an IS response over a very narrow range of salt concentrations, as well as a concomitant dependence on $\mathrm{pH}$ and temperature, which considerably limit their suitability in applications as real sensors. Another drawback is related to the time-consuming fabrication process, based on the use of hazardous substances (e.g., acrylamide) or expensive reactants.

Here, we report a PC based on a sulfonated polystyrene opal which is responsive to IS (Figure 1a). This is obtained by selfassembling of polystyrene (PS) particles followed by partial sulfonation with sulfuric acid, thereby demonstrating the possibility to produce smart photonic structures without using difficult synthetic strategies, toxic or expensive monomers, and/or etching steps. The simple fabrication method produces a hydrogel-like ordered structure, whose color can be reversibly shifted within the entire visible region $(405-760 \mathrm{~nm})$ by changing the content of water or ions over an extremely wide range of concentration (from $68 \mu \mathrm{M}$ to $4 \mathrm{M}$ ). In addition, the optical response to IS is fast (less than $1 \mathrm{~s}$ ) and selective, being mostly independent of $\mathrm{pH}$ and temperature, which makes this material very promising for sensing applications.

\section{EXPERIMENTAL SECTION}

Synthesis of Polystyrene Particles. Polystyrene particles were prepared by emulsification method starting from styrene (Sigma). In a two-necked round-bottom flask, $30 \mathrm{~mL}$ of an aqueous solution of SDS (Across) $(1 \mathrm{~g} / \mathrm{L})$ and $10 \mathrm{~mL}$ of ethanol (Sigma) were introduced. The mixture was degassed and heated at $70{ }^{\circ} \mathrm{C}$, and $3 \mathrm{~mL}$ of styrene (Sigma) was added. After vigorous stirring, $150 \mathrm{mg}$ of potassium persulfate (Across) dissolved in $10 \mathrm{~mL}$ of water was added to the mixture, and the reaction was maintained under a nitrogen atmosphere for $6 \mathrm{~h}$. The resulting white suspension of $163 \pm 3 \mathrm{~nm}$ PS particles was then passed through a filter paper and dialyzed for several days by using a cellulose membrane (Spectra/Por $7, \mathrm{MCO}=8000 \mathrm{Da})$. Solid content was about 3 wt \%.

Preparation of Sulfonated PS Opals. Opal structures were prepared by drying $7 \mathrm{~mL}$ of PS particles dispersion $3 \mathrm{wt} \%$ at $40{ }^{\circ} \mathrm{C}$ in a Petri dish $(\phi=60 \mathrm{~mm})$. In particular, $45 \mathrm{mg}$ of the resulting opal fragments was mixed in a vial with $7 \mathrm{~mL}$ of concentrated sulfuric acid (98\%, Fisher) and heated at $50{ }^{\circ} \mathrm{C}$ with an oil bath for a variable time $(1-5 \mathrm{~h})$. At the end of the reaction, the liquid phase was removed, 
and the opal fragments were abundantly washed with water, observing a progressive red-shift of the reflected opal color.

TEM Analysis. Dry-crushed (pure PS opals) and wet-crushed (sulfonated opals) particles blocks were deposited on 300 mesh carbon coated copper grids for TEM analysis. Transmission electron microscopy and EDS (energy-dispersive X-ray spectroscopy) characterization were performed on a Zeiss Libra 120 operating at $120 \mathrm{kV}$ equipped with a Bruker XFlash 6T160 SDD detector for EDS analysis and with a in-column omega filter for energy filtered imaging.

Particle Size Determination. Transmission electron microscopy images were used for the evaluation of the particles size. Quantitation was performed with the software Fiji, and the statistical analysis was carried out with the software GraphPad-Prism 5.

Measurements of the Reflected Light of Sulfonated PS Opals. Fragments of sulfonated PS opal were placed on a small coated glass-bottom dish (WillCo dish) and treated with $1.5 \mathrm{~mL}$ of solutions of varying salt concentration and composition. For each measurement of the optical response at different ionic strength, opals were extensively washed with pure water and then immersed in the salt solution, in order to ensure the exposition of the opals to right ionic strength every time. Dishes were placed on the sample holder of an inverted microscope (Zeiss AxioObserver Z1).

In order to measure the small-angle reflectivity of the opals, the microscope condenser was modified by inserting a pellicle beam splitter in the illumination path. This allowed exposing the sample to the light generated by the halogen condenser lamp and diverting a fraction of the reflected light to a lens coupled to a spectrometer (Jobin Yvon iHR550 with a Synapse CCD) through an optical fiber. To acquire the reflected-light spectrum, the condenser aperture diaphragm was closed to its minimum size in order to achieve almostcollimated sample illumination (to within $1^{\circ}$ ) and a small illumination spot (approximately $100 \mu \mathrm{m}$ ). A calibration spectrum from the light reflected by the WillCo dish glass plate was collected before each measurement session and used to normalize the spectra collected from the opals.

Angle-Resolved Reflectance Measurements. The experimental setup consisted of a collimated fiber-coupled tungsten-halogen white light source (360-2000 nm, Ocean Optics HL-2000) and a fibercoupled spectrometer (Andor Shamrock163i) connected to a CCD camera (Andor Newton CCD, cooled at $-50{ }^{\circ} \mathrm{C}$ ). The samples were placed on two rotation stages (M-060.DG, Physik Instrumente, resolution $<0.1^{\circ}$ and Thorlabs, RP01) to allow the rotation of both the sample and the collection, and we ensured that the incident light hit the crystal on the rotation axis. The reflectance spectra were collected every $5^{\circ}$, from $0^{\circ}$ up to $45^{\circ}$. All the measurements were carried out with the opal fragments totally wet in order to prevent any change in the color induced by the shrinking/swelling of the microsphere.

\section{RESULTS AND DISCUSSION}

Fabrication of Sulfonated Polystyrene Opals. The sulfonation of polystyrene opals was carried out by treating dry colloidal crystals with concentrated sulfuric acid, as previously reported by Yang et al. ${ }^{27,28}$ PS opal was obtained by evaporative deposition method on a horizontal substrate from an aqueous PS particle dispersion $(d \sim 160 \mathrm{~nm}$, see also Figure $S 1$ of the Supporting Information), resulting in a close-packed structure with the (111) plane parallel to the substrate to minimize the total repulsive energy (Figure $1 \mathrm{~b}-\mathrm{d}$ ). The sulfonation reaction carried out on self-assembled PS opals involved the external shell of each sphere, providing a well-ordered lattice of coreshell particles. Indeed, as a result of this reaction, each particle consisted of a core of highly hydrophobic polystyrene and a shell of polystyrenesulfonate (PSS), which is highly hydrophilic. As a consequence, while pristine PS opals float at the water/air interface and show poor wettability by water, the sulfonated opals are wettable and can absorb a large amount of water, thus producing a relevant volume swelling of the PC structure.
The shell thickness of PSS, which is related to the sulfonation degree, depends on several factors, such as reaction time, temperature, and particles size, as reported in the literature for nonassembled PS particles. 29,30

As the volume swelling of the PC is directly related to the PSS shell thickness, we investigated the effect of different sulfonation degrees (by using different reaction times) on the modification of the photonic architecture, with the final aim to precisely tune the IS responsive behavior. Sulfonation reactions were carried out at $50{ }^{\circ} \mathrm{C}$. However, in contrast to previous reports on PS opals sulfonation, we used much shorter reaction times $\left(1-5 \mathrm{~h}\right.$ vs $1-10$ days $^{27}$ or $\left.24 \mathrm{~h}^{28}\right)$. This choice ensured an optimum trade-off between the needs to attain a good sulfonation degree and to maintain the initial ordered arrangement of the spheres and thus the photonic effects. Interestingly, we found that such short reaction times were sufficient for altering the chemical composition of the assembled PS particles, as confirmed by infrared (IR) analysis carried out at different reaction times (see Figure S2). As the reaction time increased, we observed an enhancement of the absorbance peaks at 3460 and $1130 \mathrm{~cm}^{-1}$, characteristic of the $-\mathrm{SO}_{3} \mathrm{H}$ group. ${ }^{30}$ A simultaneous decrease of the intensity of the peaks related to the styrene rings at 756 and $700 \mathrm{~cm}^{-1}$ was observed, indicating the progressive introduction of the sulfonate group in the polymeric chains.

Morphological Characterization and Swelling Properties. As long as the sulfonation reaction proceeded, a modification of the particles surfaces was observed, not only as regards their chemical composition but also creating important changes in the opal appearance (see Figure $2 a-c$ ) and microscopic morphology. Indeed, scanning electron microscopy (SEM) analysis of the PS opal before (Figure 1d) and after sulfonation (Figure 2d,e) showed different interconnections among individual spheres. While in the pristine opal the particles boundaries were well-defined, in the sulfonated opal the particle borders were progressively less defined as long as the reaction time increased. In particular, partial interpenetration of the neighbor spheres is observed albeit the ordered PC structure is preserved, which is crucial for retaining the desired optical properties. Notably, transmission electron microscopy (TEM) analysis of the modified particles revealed that the polystyrene core dimension slightly decreased with the reaction time; a variation of about $10 \mathrm{~nm}$ in particles diameter was observed, for a pristine opal, after $3 \mathrm{~h}$ of sulfonation, (Figure $2 \mathrm{~g}-\mathrm{i}$, see also Figure S1).

Although the layer of sulfonated polystyrene was very thin (10 $\mathrm{nm}$ or less, as mentioned above), the modified opal exhibited huge swelling in water. Indeed, the introduction of sulfonic groups in the polymeric chain results in the formation of a polyelectrolytes network, in which the ionizable groups are capable of dissociation in polar media (e.g., water) with the formation of charged groups $\left(-\mathrm{SO}_{3}{ }^{-}\right)$and low molecular mass counterions $\left(\mathrm{H}^{+}\right.$or any metal cation). The presence of ionic groups along the polymer chains increases considerably the water affinity of the polymeric gel, which is able to absorb a large amount of water. As a consequence, the polyelectrolytes network undergoes a very large swelling, with a volume change that is typically 100-1000 times larger than that of an uncharged gel. ${ }^{31}$ Final swollen volume of the network is determined by the balance among different energetic contributions. Indeed, the electrostatic repulsion among the fixed charges of the polymer and the osmotic pressure of their counterions are 

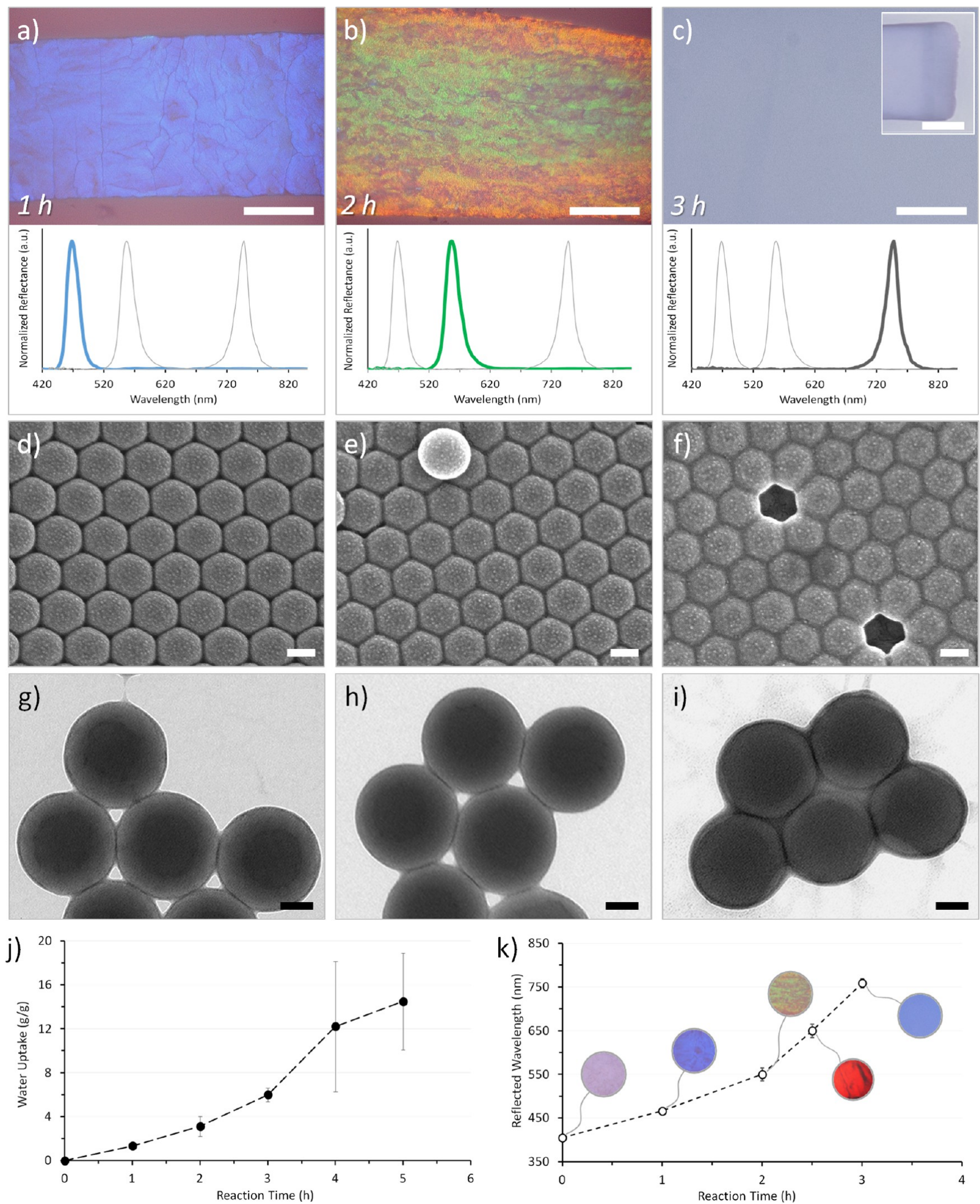

Figure 2. Optical, SEM, and TEM images of PS opals after $1 \mathrm{~h}$ (a, d, g), $2 \mathrm{~h}$ (b, e, h), and $3 \mathrm{~h} \mathrm{(c,f,i)} \mathrm{of} \mathrm{sulfonation.} \mathrm{Scale} \mathrm{bar:} 200 \mu \mathrm{m}(\mathrm{a}-\mathrm{c}), 1 \mathrm{~mm}$ (inset); $200 \mathrm{~nm}(\mathrm{~d}-\mathrm{f}) ; 50 \mathrm{~nm}(\mathrm{~g}-\mathrm{i})$. For each sulfonated opal, the reflectance spectrum is reported. Water uptake (j) and reflected peak position (k) of the sulfonated PS opals as a function of the sulfonation time. The error bars are referred to variations of the reflected wavelength among several opal fragments of the same batch. These variations are produced by little differences in the sulfonation degree of opals, which are inevitable considering the heterogeneous phase reaction between the assembled PS particles and sulfuric acid. 
counterbalanced by the energy associated with the polymer matrix elasticity. ${ }^{32,33}$

As in the case of a macroscopic gel, PS particles containing a shell of polyelectrolyte network can absorb a large amount of water and significantly increase their volume, with respect to the pristine PS one. In the sulfonated polystyrene opal, this behavior results into an important swelling of the overall photonic structure that is almost proportional to the reaction time, allowing the tuning of this property during fabrication. We measured the water uptake as a function of the reaction time (Figure 2j) as the weight ratio of absorbed water to dried opal hydrogel. As expected, the water content of the sulfonated PS opal increased with the reaction time, indicating a progressive increase of water affinity of the modified opal. Such results, in agreement with previous reports, ${ }^{27}$ showed the excellent water absorbing ability of these materials. As an example, $1 \mathrm{~g}$ of $4 \mathrm{~h}$ sulfonated PS opal was able to absorb about $10 \mathrm{~g}$ of water.

Because of the huge swelling degree of the sulfonated PS layer in water, one can assume that the shells of the particles are fused together, thus forming a continuous PSS gel network around the ordered structure of PS cores, as depicted in Figure 3a. Interpenetration of shells and formation of such a continuous gel matrix increased with the PSS thickness (sulfonation time).

Water absorption strongly influenced the mechanical properties of sulfonated opals: as expected for any hydrogel material, the opal hardness decreased with water content. In the case of a structured and discontinuous hydrogel matrix, the mechanical stability is an important aspect to take into account for easy and fast handling as well as for its deployment in sensing applications. We observed a good mechanical stability for opals with sulfonation time up to $3 \mathrm{~h}$, obtaining free-standing structures with good reflectivity which could be manipulated and could withstand repeated swelling and deswelling without compromising their structural integrity. On the other hand, by extending the reaction time to over $3 \mathrm{~h}$, very soft and fragile materials were obtained, which were could barely be handled and processed. For these reasons, we restricted the following optical characterizations to sulfonated PS opals obtained with reaction times of $1-3 \mathrm{~h}$ (see Figure S3 for $2.5 \mathrm{~h}$ sulfonated PS opals).

A direct consequence of the swelling in water of the sulfonated opal is a red-shift of the diffracted light with respect to the pristine material. In particular, as reported in Figure $2 \mathrm{k}$, the reflected wavelength gradually shifted from $405 \mathrm{~nm}$ (near$\mathrm{UV}$ ) in a pristine opal, to 470 and $550 \mathrm{~nm}$ (visible region), in the case of 1 and $2 \mathrm{~h}$ sulfonation, respectively, up to $760 \mathrm{~nm}$ (near-infrared) with $3 \mathrm{~h}$, demonstrating the ability of this method to finely tune the photonic band gap over a broad region of the electromagnetic spectrum.

Angle-Resolved Reflection Measurements. In order to investigate the optical properties of sulfonated opals, we measured the angle-resolved reflection spectra. Because the surface normal of the sample is aligned along the (111) opal plane, the maximum diffraction intensity can be obtained for wavelength $\lambda_{\max }$ obeying the Bragg-Snell equation (Figure $\left.3 \mathrm{~b}, \mathrm{c}\right):^{34}$

$$
\lambda_{\max }=\frac{2}{m} a_{[111]} \sqrt{n_{\mathrm{eff}}^{2}-\sin ^{2} \theta}=\frac{2}{m} \sqrt{\frac{2}{3}} d \sqrt{n_{\mathrm{eff}}^{2}-\sin ^{2} \theta}
$$

where $a_{[111]}$ is the interplanar spacing, $m$ is the order of Bragg diffraction, $d$ is the interparticle distance in the equilibrium state at a certain swelling condition (corresponding to $d_{0}$, diameter of pristine PS particles, in the case of FCC packed PS opals before sulfonation), $n_{\text {eff }}$ is the effective refractive index of the crystal, and $\theta$ is the angle of incidence of light with respect to the plane of the opal surface. We can also define the opal swelling degree $(S w)$ as $S w=d / d_{0}$. The effective refractive index $n_{\text {eff }}\left(n_{\text {eff }}^{2}=\varepsilon_{\text {eff }}\right.$, effective dielectric function $)$ is instead expressed by the Lorentz-Lorenz formula for the effective medium: ${ }^{35}$

$$
\frac{\varepsilon_{\text {eff }}-1}{\varepsilon_{\text {eff }}+2}=\sum \frac{\varepsilon_{i}-1}{\varepsilon_{i}+2} f_{i}
$$

where $\varepsilon_{i}$ and $f_{i}$ are the dielectric constant and the volume fraction of each $i$ portion (particles and medium), respectively.

Results of angle-resolved spectral measurements are reported in Figure $3 \mathrm{~d}-\mathrm{f}$. By plotting the dispersion of the reflectance peak as a function of the incidence angle, we obtained an excellent agreement between experimental and theoretical results, confirming that these structures behave according to the Bragg model (Figure 3f). As expected, we observed a gradual blueshift of the reflected wavelength by increasing the incidence angle with respect to normal incidence. By using the BraggSnell equation (eq 1) for fitting the data, we determined the swelling degree $S w$ and the effective refractive index $n_{\text {eff }}$ for the opals with different sulfonation degrees. In particular, we observed a progressive decrease of $n_{\text {eff }}$ with the sulfonation degree, passing from $1.53 \pm 0.03$ for $1 \mathrm{~h}$ sulfonation to $1.47 \pm$ 0.03 and $1.44 \pm 0.07$ for 2 and $3 \mathrm{~h}$, respectively. This trend indicates a decreasing contribution of the high refractive index material (PS) and a concomitant increase of the low refractive index material (hydrated PSS). On the contrary, the swelling degree increased with the sulfonation time. In fact, the $d / d_{0}$ ratio changed from $1.14 \pm 0.03(1 \mathrm{~h})$ up to $1.39 \pm 0.03(2 \mathrm{~h})$ and $1.9 \pm 0.1(3 \mathrm{~h})$, confirming a direct relationship between particle swelling and sulfonation degree. These results are consistent with the proposed model of a continuous hydrated PSS gel network embedding the ordered structure of PS cores, as it is possible verify by applying Lorentz-Lorenz formula (see eq 2) for effective refractive index. In particular, considering the obtained effective refractive indexes, given the refractive index of PS $n_{\mathrm{PS}}=1.59$ and considering the PS volumetric fractions (calculated from $d / d_{0}$ ), we obtain for the hydrated PSS gel network containing the PS particles a refractive index of $1.50,1.44$, and 1.43 (respectively for 1,2 , and 3 h sulfonation). These values are fully consistent also considering that the hydrated PSS should have a refractive index in between PS and water (water refractive index $n_{\mathrm{W}}=1.33$ ).

Optical Response Characterization. The presence of a polyelectrolyte network in these PCs introduces the possibility to modulate their optical properties by applying specific external stimuli. In particular, as in the case of hydrogel-infiltrated opals, it is possible to dynamically control the lattice constant and thus its reflected color by varying the gel volume. We triggered this type of actuation by changing the water content inside the polymeric matrix or the ionic strength of the surrounding solution, obtaining large and reversible shifts of the reflected wavelength. This response was observed for all of the sulfonated opals. However, as predictable, the maximum shift of reflected wavelengths that can be obtained depends on the sulfonation degree.

The temporal evolution of the reflection spectra of a $3 \mathrm{~h}$ sulfonated PS opal during the drying process at room temperature 
a)
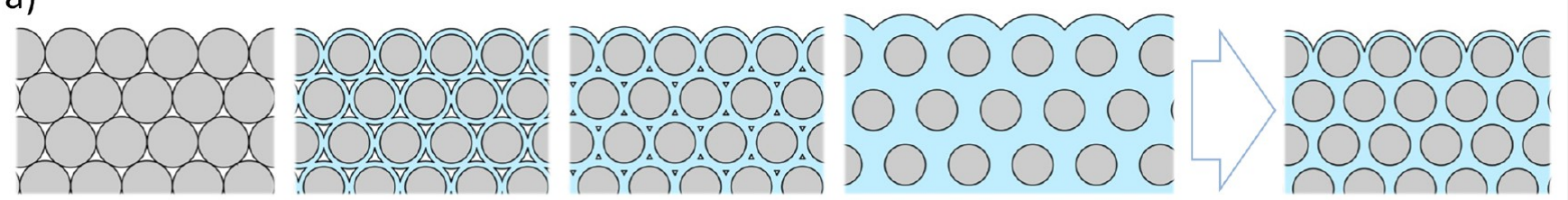

Sulfonation / Hydration

Dehydration / Deswelling

b)

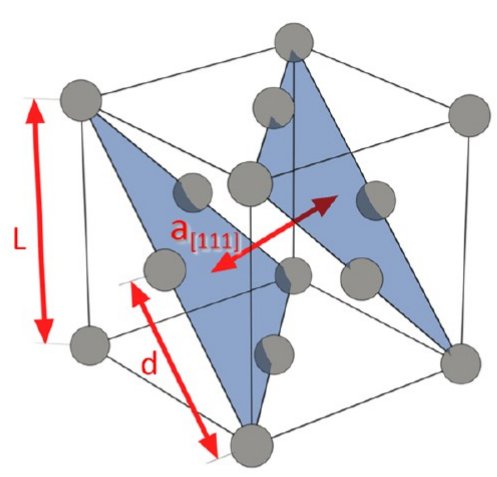

d)

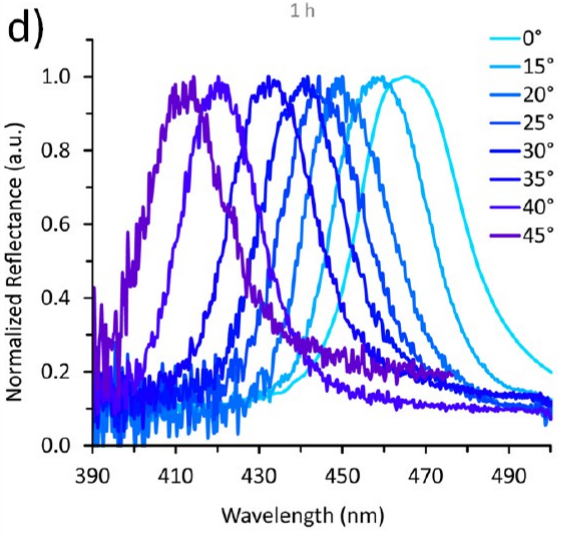

g)

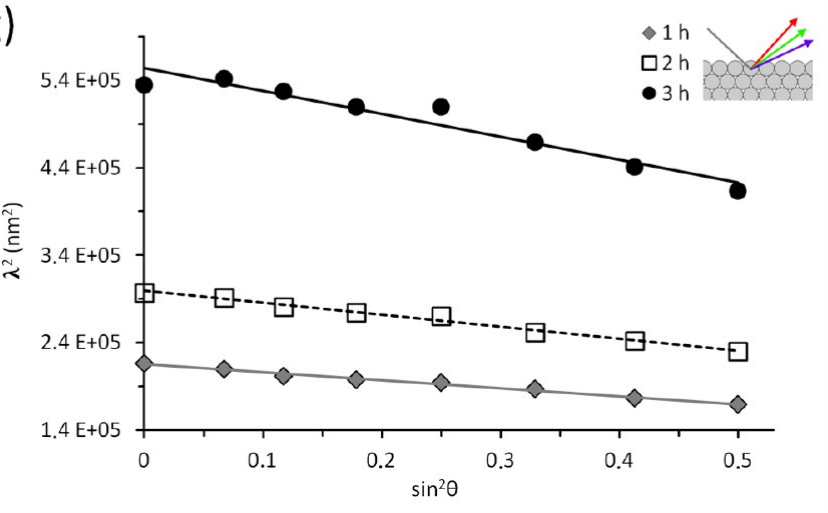

e) c)

c) $\quad \vdots[111]$
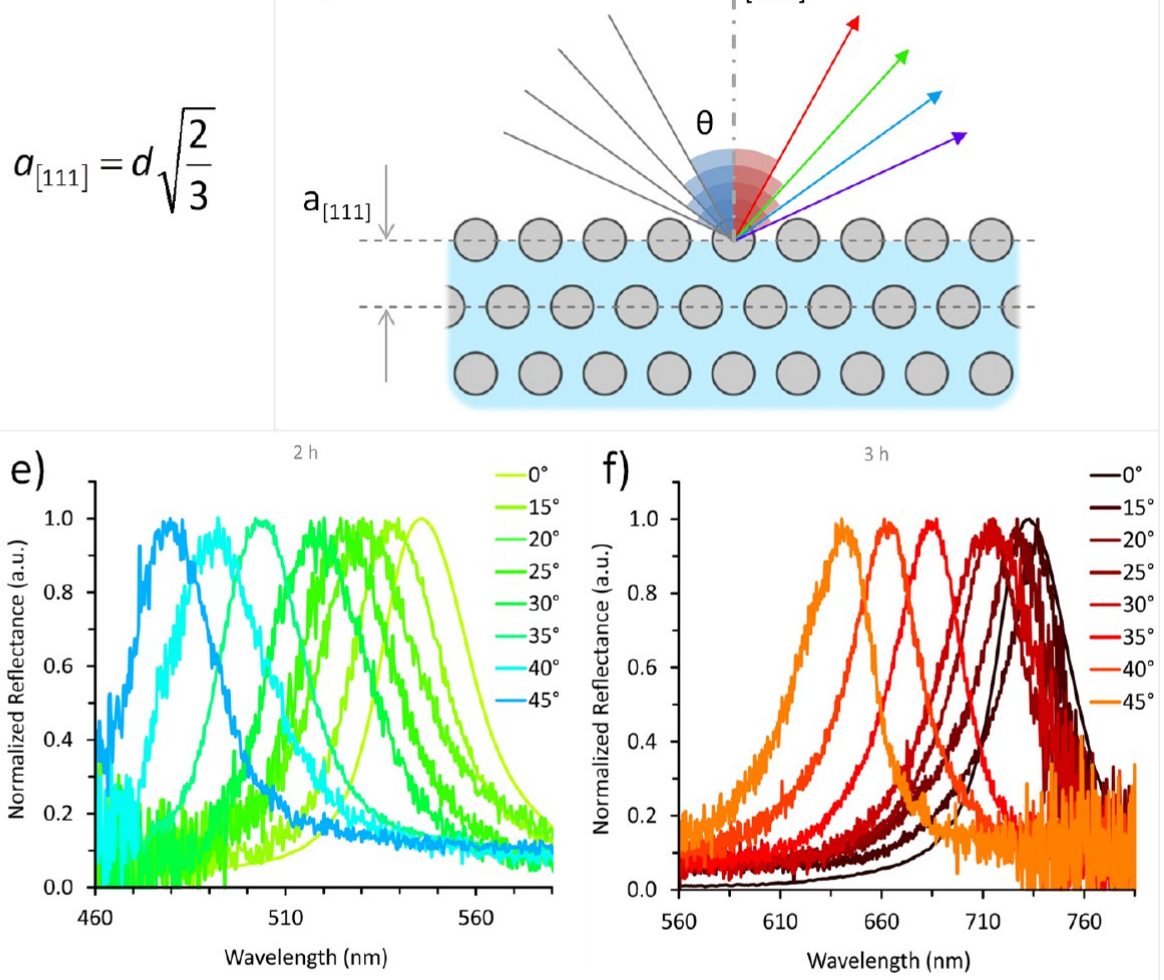

h)

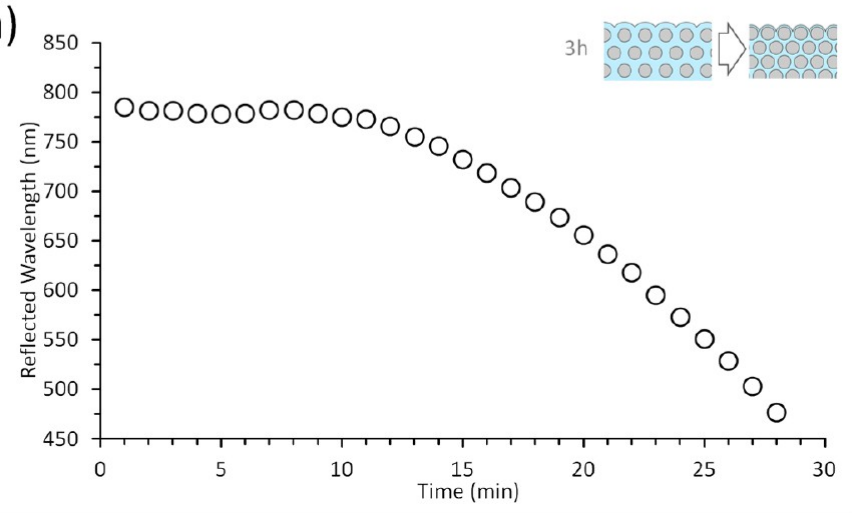

Figure 3. (a) Schematic representation of the gradual PSS shell fusion after sulfonation and hydration of PS opals. Representation of face-centered cubic packing (b) and Bragg diffraction (c). Angle-resolved reflection spectra of close-packed PS opals sulfonated for $1 \mathrm{~h}$ (d), $2 \mathrm{~h}$ (e), and $3 \mathrm{~h}$ (f). (g) Plot of squared wavelength (reflection maxima) vs $\sin ^{2} \theta$ ( $\theta$ incidence angle with respect to the normal) of PS opals sulfonated for $1 \mathrm{~h}(\bullet)$, $2 \mathrm{~h}(\square)$, and $3 \mathrm{~h}(\bullet)$. (h) Reflected peak wavelength of $3 \mathrm{~h}$ sulfonated PS opal during a dehydration process at room temperature.

is reported in Figure $3 \mathrm{~h}$. The reflected wavelength range shifted from red to blue (peak shifting from 785 to $476 \mathrm{~nm}$ ), thus covering the entire visible spectrum. Evaporation of the water contained in the opal structure induced a gradual shrinking of each particle that resulted into an "ordered collapse" of the overall structure while maintaining the photonic structure. The diffracted wavelength shift was rather slow at the beginning of the drying process, when water in excess was still stored on the surface of the structure. As the PC dried up, the evaporation started to extract the solvation water contained in the hydrated shell of the sulfonated particles, thereby producing a faster color change.

Rehydration of a partially dried sulfonated PS opal was possible by simply immersing the material in water, with full 

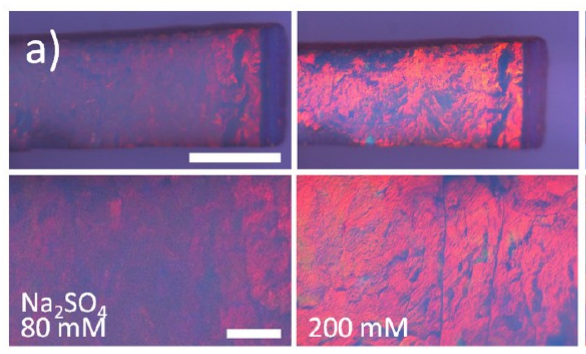

b)

b)

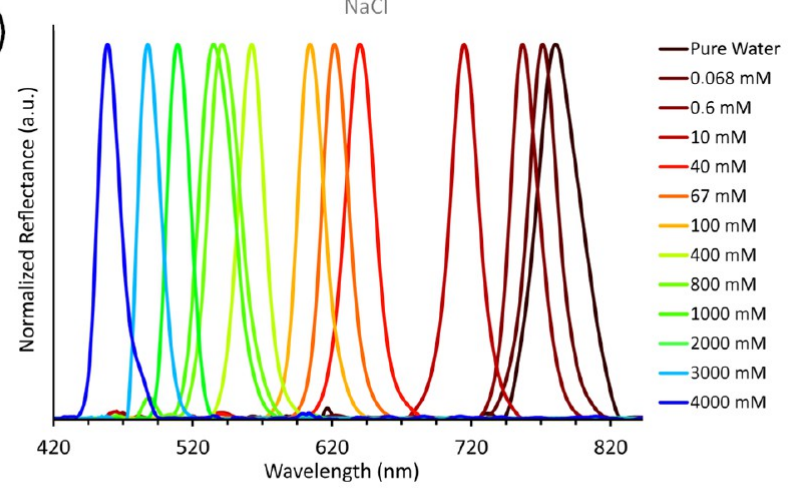

d)

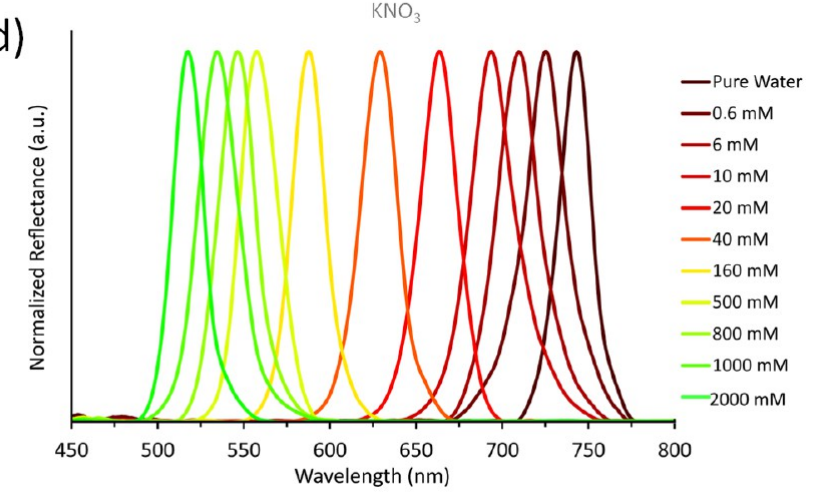

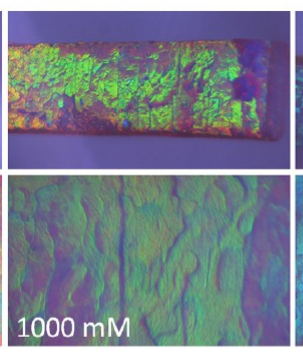
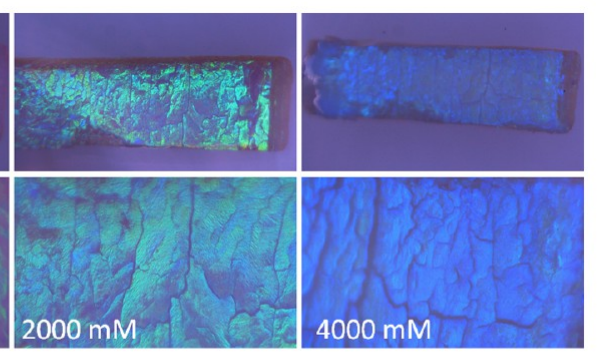

c)

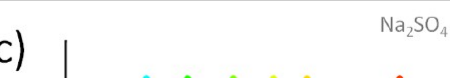

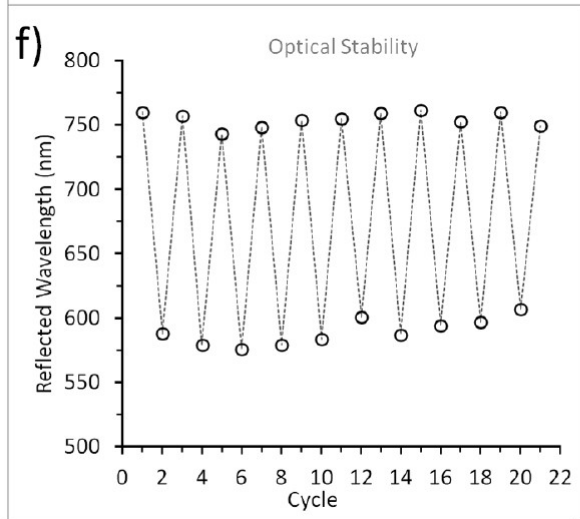
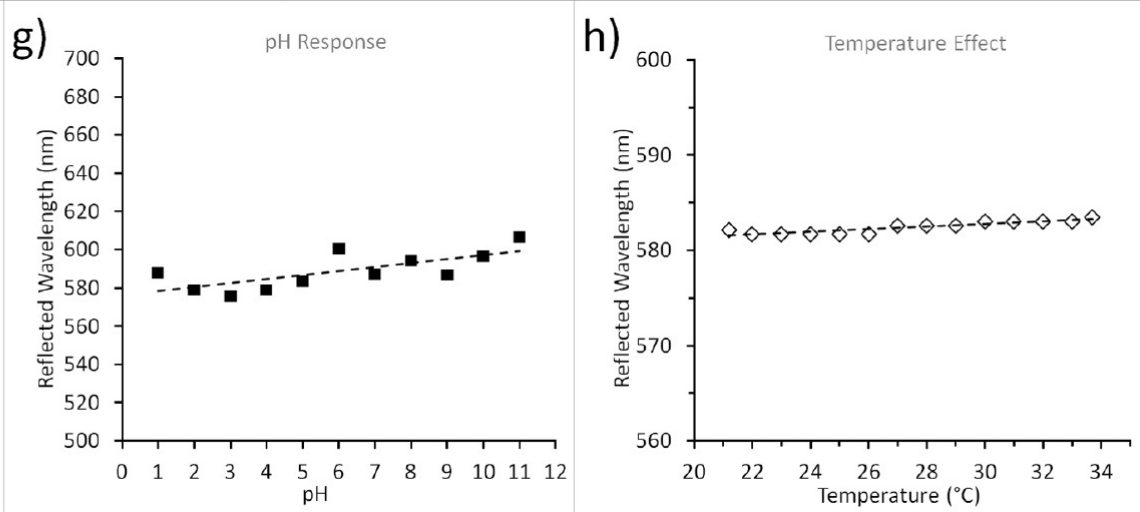

Figure 4. (a) Optical images of $3 \mathrm{~h}$ sulfonated PS opal immersed in $\mathrm{Na}_{2} \mathrm{SO}_{4}$ solutions at different IS. Scale bar: $1 \mathrm{~mm}$ (low magnification) and $200 \mu \mathrm{m}$ (high magnification). IS response of the $3 \mathrm{~h}$ sulfonated PS opal in solution of (b) $\mathrm{NaCl},(\mathrm{c}) \mathrm{Na}_{2} \mathrm{SO}_{4}$, and (d) $\mathrm{KNO}_{3}$ and (e) their corresponding reflected peak positions vs IS in logarithmic scale (linear scale in the inset). The reported concentration values represent the final ionic strength of the solution where the opals were immersed in. Optical stability (f) of $3 \mathrm{~h}$ sulfonated opal over a series of 21 cycles of pure water and a solution with a IS of $300 \mathrm{mM} . \mathrm{pH}(\mathrm{g})$ and temperature $(\mathrm{h})$ effect on the reflected peaks at constant IS by using $\mathrm{Na}_{2} \mathrm{SO}_{4}$.

recovery of the initial color within few seconds. On the other hand, in the case of completely dried sulfonated opal, the addition of water disrupted the integrity of the particles assembly, with a concomitant loss of the optical properties. This behavior can be explained by considering the different swelling degrees of particles located in different parts of the crystals due to the diffusion of the solvent from the external surface to the crystal bulk. Indeed, due to the direct contact with the solvent, the outer particles are initially subjected to a higher swelling degree than the internal ones. Since the hydration from dry state is a much faster and abrupt process respect to the rehydration from partially wet state, the expansion mismatch between wet and 
dry domains determined by the diffusion process produces a strain inside the material, which results in a fragmentation of the opal structure. On the contrary, if the opal structure is not completely dry, the rehydration can be easily carried out. In this case, in fact, the external water can freely diffuse inside the material, without causing any breakage of the assembly. As expected for a polyelectrolyte gel, the swelling degree could be reduced by the presence of salts in the surrounding solution for the creation of the Donnan equilibrium, which provides the relationship between the equilibrium concentrations of salt ions within the gel and in the surrounding solution. In particular, by increasing the salt content of the solution, the osmotic pressure in the network decreases and the hydrogel matrix tends to collapse. $^{36}$

On the basis of this behavior, we investigated how the optical properties of the sulfonated PS opals can be affected by the IS of the surrounding solution. We observed a striking change of the reflected color associated with changing IS (Figure 4a). In order to quantify the IS response, we measured the reflectance by immersing the sulfonated PS opal fragments in solutions with different salt concentrations at constant $\mathrm{pH}$ value. For this purpose, $\mathrm{NaCl}, \mathrm{KNO}_{3}$, and $\mathrm{Na}_{2} \mathrm{SO}_{4}$ were used as ionic species to vary the IS. The reflected peak wavelength could be shifted over the entire visible spectrum, from the near-infrared in pure water up to blue in highly concentrated saline solutions (Figure $4 \mathrm{~b}-\mathrm{d}$ ). At very low IS (about $70 \mu \mathrm{M}$ ) the reflected wavelength was almost insensitive with respect to the presence of ionic species, and only a shift of few nanometers was obtained. However, by increasing the IS up to $6 \mathrm{mM}$, a color change (reflected wavelength shift of about $30 \mathrm{~nm}$ ) was observed. From this point on, small variations of the IS (below about $500 \mathrm{mM}$ ) caused large color changes (wavelength shift up to $200 \mathrm{~nm}$ ). Additional increase of the IS up to $4 \mathrm{M}$ produced a smoother color shift toward the blue region. This trend was observed with all of the salts used for the IS responsive optical characterization.

By plotting the reflected peak wavelength against the IS of the surrounding solution, we observed a logarithmic relation (Figure 4e). A semilogarithmic representation enables a better appreciation that the color shift was almost independent of the salt nature and that the PC had a logarithmic IS response over an extremely broad salt concentration range. To the best of our knowledge, this is the first example of responsive PC able to change its color as a function of the IS over such a wide range of IS, thereby making these materials particularly appealing for sensing applications. ${ }^{37}$

The color change upon variation of the IS was very fast (within $1 \mathrm{~s}$ ) and fully reversible (see Figure S5 and related Video S1). Contrary to responsive PCs that can triggered by chemical stimuli, in which the response is often very slow due to the diffusion processes of the chemical species, sulfonated PS opals showed a fast response even at low IS. This resulted from the reduced dimensions of the particle assembly that ensures a rapid diffusion of the water solvent from/to the opal structure.

We tested the optical stability and absence of hysteresis by immersing an opal fragment in a solution with a IS of $300 \mathrm{mM}$ and subsequently in pure water over several cycles (Figure 4f). The initial color could be completely recovered by washing the sulfonated opal with pure water, in order to lower the IS and regain the initial swelling degree of the sulfonated PS particles. The slight variation of each cycle around a mean value is due to the moderate spatial uniformity of the sample.
In order to evaluate the influence of the $\mathrm{pH}$ on the IS-responsive behavior of the PCs, the reflectance measurements were performed by using different buffer solutions with a $\mathrm{pH}$ value in the range $1-11$ at constant IS of $300 \mathrm{mM}$. Other examples of IS responsive PCs, based on partially hydrolyzed polyacrylamide hydrogel, ${ }^{26,38}$ resulted strongly affected at $\mathrm{pH}<5$ due to the weak acidity of the carboxylic functionalities, which can act as charged groups only at $\mathrm{pH}>5$. Below this $\mathrm{pH}$ value the carboxylate groups of the polymer network are protonated and transformed into the corresponding neutral carboxylic groups, causing the loss of the IS responsiveness. On the contrary, the reflected wavelength of sulfonated PS opal resulted almost independent of the $\mathrm{pH}$ of the surrounding solution over the whole range of $\mathrm{pH}$ tested, with a shift of just about $25 \mathrm{~nm}$ (Figure $4 \mathrm{~g}$ ). This independence can be ascribed to the presence of sulfonic moieties as charged groups in the polyelectrolyte matrix. In fact, thanks to their very strong acidity, sulfonic functionalities are totally dissociated even at very low $\mathrm{pH}$ values $(\mathrm{pH}<5)$. In this way the concentration of charged units within the polymer network remains almost unvaried, assuring at any time a constant osmotic pressure and then a constant swelling degree. As a result, because the effect of $\mathrm{pH}$ on the color change can be neglected, a shift of the reflected color in sulfonated opals could be associated simply to the total concentration of the ions dissolved in the surrounding solution.

Similarly, the effect of changes in temperature on the optical response of these sulfonated PS opals was tested in the range of $21-34{ }^{\circ} \mathrm{C}$, by maintaining the IS at a constant value of $300 \mathrm{mM}$. Other ionic strength conditions were also tested obtaining similar results (data not shown). The opal color resulted to be totally independent of the temperature over the full course of heating and cooling cycles on the considered temperature range (Figure $4 \mathrm{~h}$ ).

\section{CONCLUSIONS}

In conclusion, we have developed a new type of IS responsive photonic crystals characterized by high selectivity, whose reflected color can be shifted over the entire near-IR-visible spectrum by changing the IS of the surrounding solution. The optical response is totally $\mathrm{pH}$ and temperature independent, and the initial color can be fully recovered by washing the opals with pure water. As a practical result of our investigation on how the sulfonation degree affects the swelling behavior of the self-assembled opal architecture, fine-tuning of IS responsivity and optical properties is achieved through a facile, inexpensive, and environment friendly process. These new smart photonic materials could find important technological application as all polymer sensors, with short response time, full reversibility, and easy readout. In fact, this study permits to envision application of sulfonated PS opals as inexpensive, reusable, and not photobleachable chromogenic IS sensors for environmental monitoring as well as for healthcare screening with optical readout by naked eye or by interfacing with automated optoelectronic systems.

\section{ASSOCIATED CONTENT}

\section{S Supporting Information}

The Supporting Information is available free of charge on the ACS Publications website at DOI: 10.1021/acsami.6b14455.

More details on characterization of opals (PDF)

Short movie showing the dynamic response of opal upon exposure to a water solution with variable IS (AVI) 


\section{AUTHOR INFORMATION}

\section{Corresponding Authors}

*E-mail luca.nucara@iit.it (L.N.).

*E-mail francesco.greco@iit.it (F.G.).

*E-mail virgilio.mattoli@iit.it (V.M.).

\section{ORCID}

Luca Nucara: 0000-0002-9522-1986

Francesco Greco: 0000-0003-2899-8389

Virgilio Mattoli: 0000-0002-4715-8353

Notes

The authors declare no competing financial interest.

\section{ACKNOWLEDGMENTS}

L.N. thanks Domenico Nucara and Francesca Costante for their continuous help over the years. Dr. Grazia Iannucci is thanked for her extraordinary and incessant support. This work was partially supported by the EU FP7 Marie Curie Initial Training Network CONTEST under Grant PITN-GA-2012317488 , and the H2020 ETN SYNCHRONICS under Grant 643238. F.C. is a Royal Society Wolfson Research Merit Award holder.

\section{REFERENCES}

(1) Di Stasio, F.; Berti, L.; McDonnell, S. O.; Robbiano, V.; Anderson, H. L.; Comoretto, D.; Cacialli, F. Fluorescent Polystyrene Photonic Crystals Self-Assembled with Water-Soluble Conjugated Polyrotaxanes. APL Mater. 2013, 1 (4), 042116.

(2) Couturier, J.-P.; Sütterlin, M.; Laschewsky, A.; Hettrich, C.; Wischerhoff, E. Responsive Inverse Opal Hydrogels for the Sensing of Macromolecules. Angew. Chem., Int. Ed. 2015, 54, 6641-6644.

(3) Ahn, Y.; Kim, E.; Hyon, J.; Kang, C.; Kang, Y. Photoresponsive Block Copolymer Photonic Gels with Widely Tunable Photosensitivity by Counter-Ions. Adv. Mater. 2012, 24 (23), OP127-OP130.

(4) Wang, J. Y.; Cao, Y.; Feng, Y.; Yin, F.; Gao, J. P. Multiresponsive Inverse-Opal Hydrogels. Adv. Mater. 2007, 19 (22), 3865-3871.

(5) Hong, W.; Hu, X.; Zhao, B.; Zhang, F.; Zhang, D. Tunable Photonic Polyelectrolyte Colorimetric Sensing for Anions, Cations and Zwitterions. Adv. Mater. 2010, 22 (44), 5043-5047.

(6) Fenzl, C.; Hirsch, T.; Wolfbeis, O. S. Photonic Crystals for Chemical Sensing and Biosensing. Angew. Chem., Int. Ed. 2014, 53 (13), 3318-3335.

(7) Ma, H.; Zhu, M.; Luo, W.; Li, W.; Fang, K.; Mou, F.; Guan, J. Free-Standing, Flexible Thermochromic Films Based on One-Dimensional Magnetic Photonic Crystals. J. Mater. Chem. C 2015, 3 (12), $2848-2855$

(8) Sugiyama, H.; Sawada, T.; Yano, H.; Kanai, T. Linear Thermosensitivity of Gel-Immobilized Tunable Colloidal Photonic Crystals. J. Mater. Chem. C 2013, 1 (38), 6103.

(9) Chen, M.; Zhou, L.; Guan, Y.; Zhang, Y. Polymerized Microgel Colloidal Crystals: Photonic Hydrogels with Tunable Band Gaps and Fast Response Rates. Angew. Chem., Int. Ed. 2013, 52 (38), 99619965.

(10) Nucara, L.; Greco, F.; Mattoli, V. Electrically Responsive Photonic Crystals: A Review. J. Mater. Chem. C 2015, 3, 8449-8467.

(11) He, L.; Wang, M.; Ge, J.; Yin, Y. Magnetic Assembly Route to Colloidal Responsive Photonic Nanostructures. Acc. Chem. Res. 2012, 45 (9), 1431-1440.

(12) Hu, H.; Chen, C.; Chen, Q. Magnetically Controllable Colloidal Photonic Crystals: Unique Features and Intriguing Applications. J. Mater. Chem. C 2013, 1 (38), 6013.

(13) Chan, E. P.; Walish, J. J.; Urbas, A. M.; Thomas, E. L. Mechanochromic Photonic Gels. Adv. Mater. 2013, 25 (29), 39343947.

(14) Schäfer, C. G.; Winter, T.; Heidt, S.; Dietz, C.; Ding, T.; Baumberg, J. J.; Gallei, M. Smart Polymer Inverse-Opal Photonic
Crystal Films by Melt-Shear Organization for Hybrid Core-shell Architectures. J. Mater. Chem. C 2015, 3, 2204-2214.

(15) Liu, H.-Y.; Wang, C.-T.; Hsu, C.-Y.; Lin, T.-H.; Liu, J.-H Optically Tuneable Blue Phase Photonic Band Gaps. Appl. Phys. Lett. 2010, 96 (12), 121103.

(16) Zhao, J.; Liu, Y.; Yu, Y. Dual-Responsive Inverse Opal Films Based on a Crosslinked Liquid Crystal Polymer Containing Azobenzene. J. Mater. Chem. C 2014, 2 (48), 10262-10267.

(17) Kamenjicki, M.; Lednev, I. K.; Asher, S. A. Photoresponsive Azobenzene Photonic Crystals. J. Phys. Chem. B 2004, 108 (34), 12637-12639.

(18) Ge, J.; Yin, Y. Responsive Photonic Crystals. Angew. Chem., Int. Ed. 2011, 50 (7), 1492-1522.

(19) Burgess, I.; Lončar, M.; Aizenberg, J. Structural Colour in Colourimetric Sensors and Indicators. J. Mater. Chem. C 2013, 1 (38), 6075-6086.

(20) Ye, B.-F.; Zhao, Y.-J.; Cheng, Y.; Li, T.-T.; Xie, Z.-Y.; Zhao, X.W.; Gu, Z.-Z. Colorimetric Photonic Hydrogel Aptasensor for the Screening of Heavy Metal Ions. Nanoscale 2012, 4 (19), 5998-6003.

(21) Holtz, J. H.; Asher, S. A. Polymerized Colloidal Crystal Hydrogel Films as Intelligent Chemical Sensing Materials. Nature 1997, 389 (6653), 829-832.

(22) Holtz, J. H.; Holtz, J. S. W.; Munro, C. H.; Asher, S. A. Intelligent Polymerized Crystalline Colloidal Arrays: Novel Chemical Sensor Materials. Anal. Chem. 1998, 70 (4), 780-791.

(23) Zhang, J. T.; Wang, L.; Luo, J.; Tikhonov, A.; Kornienko, N.; Asher, S. A. 2-D Array Photonic Crystal Sensing Motif. J. Am. Chem. Soc. 2011, 133 (24), 9152-9155.

(24) Lee, Y.-J.; Braun, P. V. Tunable Inverse Opal Hydrogel pH Sensors. Adv. Mater. 2003, 15 (78), 563-566.

(25) Lee, K.; Asher, S. A. Photonic Crystal Chemical Sensors: pH and Ionic Strength. J. Am. Chem. Soc. 2000, 122 (14), 9534-9537.

(26) Fenzl, C.; Wilhelm, S.; Hirsch, T.; Wolfbeis, O. S. Optical Sensing of the Ionic Strength Using Photonic Crystals in a Hydrogel Matrix. ACS Appl. Mater. Interfaces 2013, 5 (1), 173-178.

(27) Yang, Z.; Li, D.; Rong, J.; Yan, W.; Niu, Z. Opal Hydrogels Derived by Sulfonation of Polystyrene Colloidal Crystals. Macromol. Mater. Eng. 2002, 287, 627-633.

(28) Niu, Z. W.; Li, D.; Yang, Z. Z.; Hu, Z. B.; Lu, Y. F.; Han, C. C. Responsive Binary Composite Hydrogel Opals. ChemPhysChem 2003, 4 (8), 865-868.

(29) Reese, C.; Guerrero, C.; Weissman, J.; Lee, K.; Asher, S. A. Synthesis of Highly Charged, Monodisperse Polystyrene Colloidal Particles for the Fabrication of Photonic Crystals. J. Colloid Interface Sci. 2000, 232 (1), 76-80.

(30) Hazarika, M.; Malkappa, K.; Jana, T. Particle-Size-Dependent Properties of Sulfonated Polystyrene Nanoparticles. Polym. Int. 2012, 61 (9), 1425-1432.

(31) Hua, J.; Mitra, M. K.; Muthukumar, M. Theory of Volume Transition in Polyelectrolyte Gels with Charge Regularization. J. Chem. Phys. 2012, 136 (13), 134901.

(32) Flory, P. J. Principles of Polymer Chemistry; Cornell University Press: Ithaca, NY, 1953.

(33) Quesada-Pérez, M.; Maroto-Centeno, J. A.; Forcada, J.; HidalgoAlvarez, R. Gel Swelling Theories: The Classical Formalism and Recent Approaches. Soft Matter 2011, 7 (22), 10536.

(34) Saito, H.; Takeoka, Y.; Watanabe, M. Simple and Precision Design of Porous Gel as a Visible Indicator for Ionic Species and Concentration. Chem. Commun. 2003, 81 (17), 2126-2127.

(35) Morandi, V.; Marabelli, F.; Amendola, V.; Meneghetti, M.; Comoretto, D. Colloidal Photonic Crystals Doped with Gold Nanoparticles: Spectroscopy and Optical Switching Properties. Adv. Funct. Mater. 2007, 17 (15), 2779-2786.

(36) Kramarenko, E. Y.; Philippova, O. E.; Khokhlov, A. R. Polyelectrolyte Networks as Highly Sensitive Polymers. Polym. Sci., Ser. C 2006, 48 (1), 1-20.

(37) To further investigate the cation effect, we carried out some experiments by using $\mathrm{Ca}^{2+}$ salt solutions at different concentrations. However, contrary to the results obtained with monovalent cations, 
calcium ions produced large shifts of the reflected wavelength at lower concentration than sodium or potassium salts. Preliminary results are reported in the Supporting Information (see Figure S4).

(38) Xu, X.; Goponenko, A. V.; Asher, S. A. Polymerized polyHEMA Photonic Crystals: $\mathrm{pH}$ and Ethanol Sensor Materials. J. Am. Chem. Soc. 2008, 130 (30), 3113-3119. 